\title{
Spread of CTX-M-type ESBLs in isolates of E. coli from long-term care and rehabilitation facilities in Northern Italy
}

\author{
Elisabetta Nucleo', Roberta Migliavacca', Michela Balzaretti', Fabiola Martino', \\ Melissa Spalla ${ }^{3}$, Cristina Terulla ${ }^{3}$, Laura Pagani' \\ 'Università di Pavia, \\ ${ }^{2}$ Laboratorio di Microbiologia ASP Piero Redaelli, Milano, \\ ${ }^{3}$ Laboratorio di Microbiologia IRCCS S. Matteo, Pavia
}

Key words: CTX-M, E. coli, LTCRF, epidemic diffusion

Diffusione di Extended-Spectrum-ß-Lattamasi di tipo CTX-M in isolati clinici di E. coli provenienti da strutture di lungodegenza e riabilitazione geriatriche del Nord Italia

\section{SUMMARY}

During the period March 2003 - May 2004 at the Laboratory of Clinical Microbiology "Redaelli" LTCRF in Milan, Italy, a total of $529 \mathrm{E}$. coli, obtained from inpatients of 3 different Long Term Care Rehabilitation Facilities (LTCRFs) in Northern Italy, were processed and 77 ESBLs producers (14.5\%) were identified by Vitek System. The results were confirmed by double-disk synergy test with tazobactam (TZP). 6I/77 isolates were characterized by higher levels of resistance to cefotaxime (CTX) than to ceftazidime (CAZ). (B-lactamase production was investigated by analytical isoelectric focusing (IEF) coupled with a bioassay and showed multiple (B-lactamase bands including one enzyme with pl 8.4 that, in a bioassay, was more active on CTX, ATM than on CAZ. The presence of (B-lactamase genes was investigated by colony blot hybridization and by PCR amplification of blaTEM, blaSHV and blaCTX-M alleles. 43/6I isolates produced both TEM-I and CTX-M-type enzymes, I4/6I expressed only CTX-M-type while in 4 cases were found blaCTX-M, blaTEM and blaSHV genes. The remainders (16/77), characterized by high levels of resistance to both CTX and CAZ, produced TEM-I and SHV-5 enzymes (1/16) and TEM type ESBLs (15/16). Conjugation experiments, performed in liquid medium, confermed that the ESBLs determinants were transferable. Pulsed-field gel electrophoresis profiles of genomic DNA, digested with Notl, were analysed and revealed clonal heterogeneity.

Our work confirms the emergence of CTX-M-type enzymes and their spread in Northern Italy also in longterm care and rehabilitation facilities that may be an important reservoir of ES?L producing E. coli.

Received June 8, 2006

Accepted April 16, 2008

\section{INTRODUZIONE}

La produzione di Extended-Spectrum $\beta$-lattamasi (ESßLs) negli enterobatteri, rappresenta un problema di rilevante impatto clinico ed epidemiologico che, recentemente, si è esteso anche alle strutture di lungodegenza riabilitativa (LTCRF) ed alle case di cura per anziani $(9,12,2)$.

In queste strutture, che accolgono una popolazione caratterizzata da numerosi fattori di rischio predisponenti all'acquisizione di infezioni nosocomiali, è spesso necessario ricorrere a terapie antibiotiche intense e prolungate che favoriscono la selezione dei determinanti di resistenza.

La somministrazione indiscriminata di cefalospo- rine a spettro allargato è una pratica ricorrente all'interno di queste strutture, in cui vengono isolati frequentemente microrganismi produttori di ESßL spesso caratterizzati da una diffusione di tipo epidemico. Il numero considerevole di pazienti sovente spostati tra diverse unità di cura alimenta, inoltre, la diffusione di tali resistenze e ne rende più difficile il controllo.

Nelle Enterobacteriaceae stanno emergendo ESßL di tipo CTX-M, enzimi la cui diffusione, sia tra batteri appartenenti a specie diverse che in differenti parti del mondo, dal 1995 ad oggi, è in drammatico aumento (4).

La prima ESßL di tipo CTX-M (FEC-1) è stata

Corresponding author: Laura Pagani

Via Brambilla 74, 27100 Pavia

Tel.: 03825484206 - Fax: 03825484255 - E-mail: Ipagani@unipv.it 
segnalata agli inizi degli anni '80. Gli enzimi di tipo CTX-M rappresentano un gruppo di ß-lattamasi a spettro esteso di classe A plasmide mediate. Humeniuk, et al. hanno identificato come progenitore delle ESßL di tipo CTX-M l'enzima cromosomico AmpC di Kluyvera ascorbata, un batterio enterico di raro riscontro clinico (1). Gli enzimi di tipo CTX-M sono caratterizzati da una maggiore attività idrolitica verso il cefotaxime (CTX), da cui il nome CTX-M, ed il ceftriaxone, piuttosto che verso il ceftazidime (CAZ); mostrano una maggiore sensibilità al tazobactam (TZP) rispetto al clavulanato (11). Il rapido sviluppo della famiglia CTX-M ha coinvolto diverse specie di Enterobacteriaceae diffuse per lo più in tre aree geografiche: Sud America, Europa ed Estremo Oriente. Recentemente sono state segnalate anche in Italia $(6,7)$. I geni blaCTX-M possono essere localizzati a livello cromosomico, ma molto spesso vengono identificati a livello plasmidico (11).

Scopo del lavoro è stato studiare la diffusione di E. coli produttori di ESßL di tipo CTX-M in tre strutture di lungodegenza riabilitativa geriatriche (LTCRFs) del Nord Italia.

\section{MATERIALI E METODI Stipiti batterici}

Nel periodo marzo 2003 - maggio 2004 sono stati raccolti, da urine di pazienti con catetere vescicale ricoverati in diverse LTCRF del Nord Italia, 77 isolati clinici di E. coli consecutivi e non replicati con fenotipo di resistenza suggestivo della produzione di ESßL.

\section{Test di sensibilità}

La sensibilità agli antibiotici e la produzione di ESßL sono state valutate in un primo tempo mediante GNS CARD (Vitek System, bioMérieux). La produzione di ESßL è stata poi confermata tramite test del doppio disco.

Tale test è stato eseguito su Mueller-Hinton (MH) agar ponendo, dopo semina a confluenza di una sospensione batterica avente torbidità pari a $0.5 \mathrm{McF}$ arland, dischetti di FEP, CAZ, CTX, ed ATM ad una distanza di $25 \mathrm{~mm}$ centro-centro da uno di TZP. La positività al test è stata attribuita sulla base della presenza di evidenti distorsioni degli aloni di inibizione verso il dischetto centrale (effetto sinergico).

\section{Caratterizzazione delle $\boldsymbol{\beta}$-lattamasi}

Da ogni ceppo in esame sono stati ottenuti estratti enzimatici grezzi. Le colture in fase logaritmica di crescita sono state centrifugate e le cellule, lavate con tampone sodio-fosfato $10 \mathrm{mM}(\mathrm{pH}$ 7.0), sono state sottoposte a sonicazione.

L'estratto enzimatico grezzo ottenuto è stato quindi sottoposto ad isoelettrofocalizzazione (IEF) in gel di poliacrilamide anfolinizzato. Le bande ßlattamasiche sono state visualizzate utilizzando una cefalosporina cromogena (nitrocefin). Il profilo di attività sui substrati delle bande beta-lattamasiche ottenute è stato determinato tramite saggio biologico: dopo IEF il gel è stato ricoperto con un sottile strato di $\mathrm{MH}$ agar addizionato di $1 \mu \mathrm{g} / \mathrm{ml}$ dello specifico antibiotico da valutare. Dopo due ore di incubazione a $37^{\circ} \mathrm{C}$, sull'agar contenente antibiotico è stata seminata a confluenza una brodocoltura cresciuta overnight di $E$. coli ATCC $25922\left(10^{8} \mathrm{UFC} / \mathrm{ml}\right)$. La crescita di $E$. coli ATCC 25922, sensibile alla concentrazione di antibiotico presente nel terreno, può avvenire e risulta visibilmente evidente solo in corrispondenza delle bande enzimatiche al cui livello il substrato in esame viene idrolizzato. In assenza di idrolisi la crescita batterica risulta inibita.

\section{Esperimenti di coniugazione}

Il trasferimento dei determinanti di resistenza è stato ottenuto in terreno liquido usando come

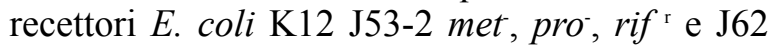
pro $^{-}$, his $^{-}$, trp, lac $^{-}, \mathrm{sm}^{r}$. I batteri transconiuganti sono stati selezionati in McConkey agar, addizionato di rifampicina $(100 \mathrm{mg} / \mathrm{l})$ o streptomicina $(1000 \mathrm{mg} / \mathrm{l})$ e cefotaxime $(2 \mathrm{mg} / \mathrm{l})$.

\section{Analisi del DNA plasmidico}

Il DNA plasmidico è stato estratto e purificato mediante lisi alcalina. Gli estratti di DNA plasmidico sono stati successivamente sottoposti a digestione enzimatica con EcoRI e quindi valutati per il profilo di restrizione.

Ricerca dei geni bla $\boldsymbol{a}_{\mathrm{TEM}}, \boldsymbol{b l a}_{\mathrm{SHV}}$ e bla $\boldsymbol{a}_{\mathrm{CTX}-\mathrm{M}}$

La ricerca dei determinanti di resistenza bla тем,

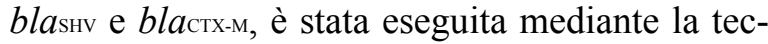
nica dell'ibridazione su colonia e l'amplificazione genica (PCR). L'ibridazione su colonia è stata eseguita su filtri di nitrocellulosa sterili posti in piastre di $\mathrm{MH}$ agar. Le sonde di ibridazione sono state sintetizzate usando il kit "ECL Randomprime direct nucleic acid labelling and detection system" (Amersham Biosciences). La rivelazione del segnale di chemiluminescenza è stata ottenuta a seguito di esposizione ad una lastra autoradiografica. Le sonde utilizzata per l'esperimento di ibridazione sono state costruite sulla base dei geni bla $_{\text {Tем, }}$ bla $a_{\text {SHV }}$ e bla СтХ-м $_{\text {с }}$ e con i seguenti primer: TEM/F- ATA AAA TTC TTG AAG AC e TEM/R

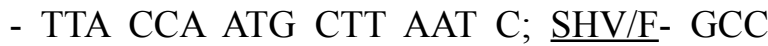
CGG GTT ATT CTT ATT TGT CGC e $\underline{\mathrm{SHV} / \mathrm{R}}$ TCT TTC CGA TGC CGC CGC CAG TCA; CTX-M-3/F- AT GGT TAA AAA ATC ACT GCG CCA G e CTX-M-3/R- TTA CAA ACC GTC GGT GAC GAT TTT. Le stesse sequenze oligonucleotidiche sono state impiegate anche per l'amplificazione, tranne che nel caso dei determi- 
nanti di tipo CTX-M, per i quali è stata utilizzata a tale scopo la coppia di primer: CTX-MU1 5'ATGTGCAGYACCAGTAARGT-3'e CTX-MU2 3'-AGACCASTGRATRAARTGGGT-5'.

Per entrambi gli esperimenti sono stati inclusi: come controllo negativo E. coli ATCC 25922, e come controlli positivi ceppi di riferimento produttori degli enzimi TEM-9, SHV-5 e di tipo CTX-M.

\section{Tipizzazione molecolare tramite PFGE (Pulsed Field Gel Electrophoresis)}

Gli isolati di E. coli sono stati tipizzati a livello molecolare utilizzando il kit di reagenti $n^{\circ} 2$ Gene Path (Bio-Rad). Il taglio di restrizione del DNA genomico ottenuto è stato realizzato mediante l'endonucleasi di restrizione NotI. I campioni sono stati sottoposti ad elettroforesi in campo pulsato utilizzando l'apparato GenePath Bio-Rad. Le relazioni clonali sono state interpretate secondo $\mathrm{i}$ criteri proposti da Tenover, et al (10).

\section{RISULTATI}

Durante il periodo Marzo 2003-Maggio 2004 sono stati raccolti, presso il Laboratorio di Microbiologia Clinica dell'ASP "Piero Redaelli" di Milano, un totale di 529 campioni clinici di E. coli. Tutti i campioni provenivano da urine di pazienti portatori di catetere vescicale. $77 / 529(14.5 \%)$ isolati sono risultati $E S ß L$ produttori sia mediante metodo automatizzato (Vitek System) che con il test del doppio disco. Gli ESßL produttori risultavano sempre multiresistenti, ma sensibili all'associazione piperacillina/tazobactam. 61/77 (79.22\%) isolati di $E$. coli $\mathrm{ESBL}$ positivi erano caratterizzati da più alti livelli di resistenza al CTX che al CAZ. L'IEF ha rivelato la presenza di bande $\beta$-lattamasiche multiple tra cui quella corrispondente ad un enzima con pI 8.4 che, con il saggio biologico, mostrava un'attività maggiore verso il CTX e l'ATM che verso il CAZ (figura I). L'ibridazione su colonia effettuata per lo screening dei determinanti di resistenza di tipo CTX$\mathrm{M}$, ha prodotto risultati positivi per tutti i 61 isolati. Gli esperimenti di PCR, eseguiti su parte dei 61 isolati positivi all'ibridazione, hanno confermato la specificità del metodo di screening, fornendo un prodotto di amplificazione delle dimensioni attese $(\sim 593 \mathrm{bp})$. L'ibridazione su colonia è stata effettuata, su tutti i ceppi CTX-M produttori, anche per la ricerca dei determinanti bla тем e blasнv. I risultati hanno indicato che 9/61 ceppi esprimevano una sola $\beta$ lattamasi di tipo CTX-M mentre 52/61 isolati presentavano bande $\beta$-lattamasiche multiple. Più precisamente, 29/52 isolati producevano contemporaneamente enzimi di tipo CTX-M e
TEM-1, 7/52 esprimevano enzimi di tipo CTX$M$ ed SHV ed, infine, in 16/52 casi erano rilevabili geni bla стх-м, bla $a_{\text {тем }}$ e blasнv. Nei restanti 16/77 ceppi, caratterizzati fenotipicamente da alti livelli di resistenza sia al CTX che al CAZ, 15 erano produttori di una $\mathrm{ES} \beta \mathrm{L}$ di tipo TEM, mentre un solo campione era produttore di una TEM-1 e di una SHV-5. La produzione di ß-lattamasi di tipo CTX-M è risultata essere mediata, in 21/61 casi, da plasmidi coniugativi.

L'analisi dei pattern di restrizione ottenuti con PFGE degli isolati CTX-M produttori ha rivelato eterogeneità clonale, con quattro linee maggiori (A-D) ed un cluster epidemico specifico per ogni struttura (figura II). I cloni A e B, responsabili di due distinti eventi epidemici in due strutture differenti, sono stati ritrovati anche coesistere all'interno di una stessa LTCRF (tabella 1). Allo stesso modo il clone (D) è stato rilevato in due differenti LTCRF, in una delle quali era presente, contemporaneamente il clone C. Nei ceppi CTX-M produttori sono stati evidenziati plasmidi coniugativi di dimensioni (comprese tra le tra le 50 e le $55 \mathrm{~Kb}$ ) e profilo di restrizione differenti, anche nell'ambito di un medesimo pulsotipo.

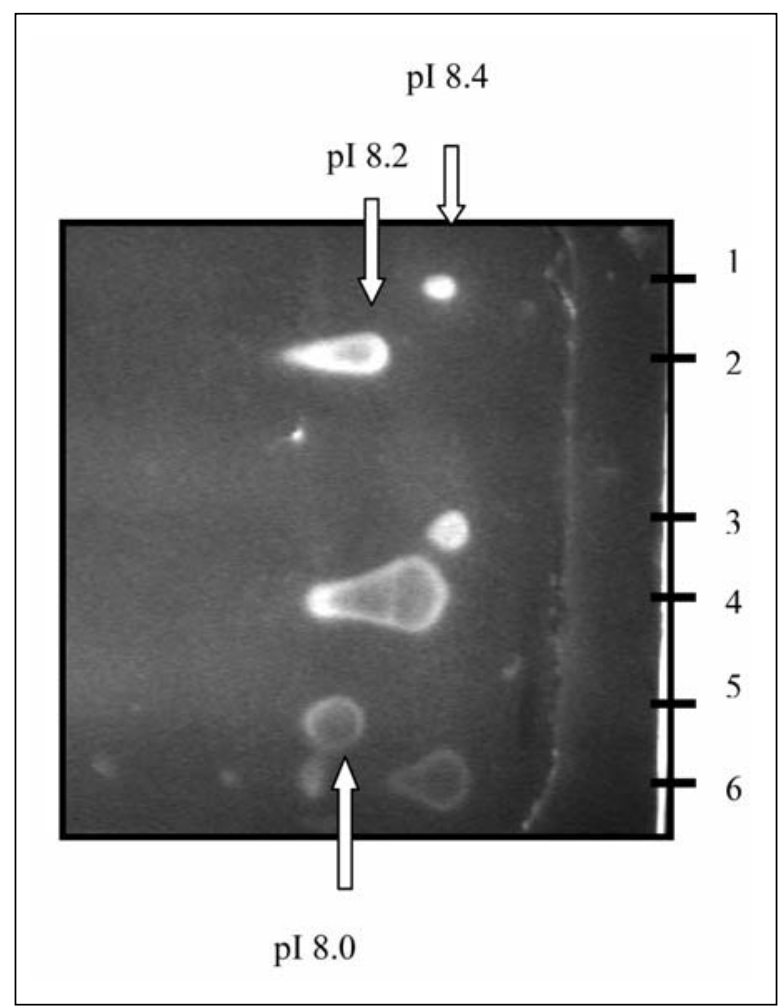

Figura I. Utilizzo della tecnica del substrato ricoprente per la rivelazione dell'attività idrolitica di estratti enzimatici grezzi di $\mathrm{E}$. coli verso il cefotaxime.

Linee da I a 6: Ceppi ST225 con pl 8.4, SHV-5 con pl 8.2, DON 130 con pl 8.4, BE II 7 con pl 8.3,TM2I5 con pl 8.0 e DVAIO con pl 8.4 e 8.0 . 


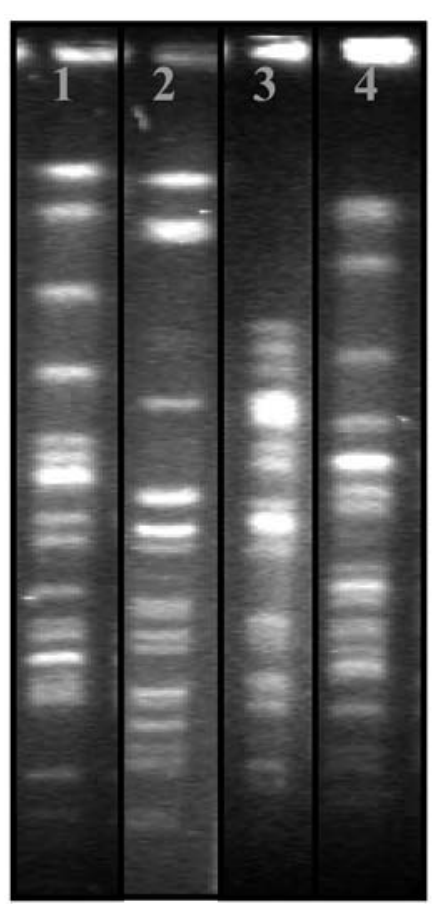

Figura II. Profili PFGE (Not I) delle quattro maggiori linee clonali, relative agli isolati clinici di E.coli CTX-M produttori. Linee da I a 4: profili A (ceppo DAG I 20), B (ceppo LG2 I 2), C (ceppo ST225), D (ceppo MR204), rispettivamente.

\section{CONCLUSIONI}

Nell'ambito della vasta famiglia delle Enterobacteriaceae, la resistenza mediata dalle ß-lattamasi ad ampio spettro di classe A rappresenta ormai un problema di crescente impatto clinico anche in Italia $(5,8)$. Tra le ESßL di classe A, gli enzimi di tipo CTX-M, in particolare, stanno diffondendosi rapidamente; in Italia tali enzimi erano stati fino ad ora riscontrati solo raramente in ambito nosocomiale e comunitario $(3,7)$. I risultati ottenuti mostrano, tuttavia, come le strutture geriatriche di riabilitazione e di lungodegenza, rappresentino un reservoir di E. coli CTX-M

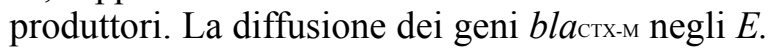
coli isolati è risultata essere sia plasmide - che clone- mediata. Pertanto, la diffusione di questi geni di resistenza, sembra destinata, sul territorio nazionale, ad aumentare. Sarebbe auspicabile, perciò, per arginare il problema della diffusione di enterobatteri ESßL-produttori, l'attuazione di un maggior numero di efficaci misure di controllo. Tali accorgimenti potrebbero consistere nel monitoraggio dei pazienti al ricovero nonché del controllo periodico dei portatori di cateteri vescicali potenzialmente colonizzati o infettati da microrganismi antibiotico-resistenti.

Tabella I. Diffusione di cloni di E. coli CTX-M produttori nelle tre strutture di lungodegenza riabilitativa geriatriche

\begin{tabular}{lccc}
\hline & Istituto Golgi & Ospedale geriatrico Redaelli & Ospedale geriatrico Redaelli \\
& (Abbiategrasso) & (Vimodrone) & (Milano) \\
\hline Pulsotipi & $\mathrm{D}, \mathrm{B},{ }^{*} \mathrm{C}$ & $* \mathrm{~A}, \mathrm{~B},{ }^{* \mathrm{D}}$ & $* \mathrm{~B}$ \\
& ed altri sporadici & ed altri sporadici & ed altri sporadici \\
\hline
\end{tabular}

*clone responsabile di un evento epidemico all'interno della struttura ospedaliera.

\section{BIBLIOGRAFIA}

1. Bonnet R. Growing Group of Extended-Spectrum BLactamases: the CTX-M Enzymes. Antimicrob Agents Chemother 2004; 48: 1-14.

2. Bradford PA, Cherubin CE, Idemyor V, Rasmussen BA, Bush K. Multiply resistant Klebsiella pneumoniae strains from two Chicago Hospitals: identification of the extended-spectrum TEM-12 and TEM-10 ceftazidime-hydrolyzing ß-lactamases in a single isolate. Antimicrob Agents Chemother 1994; 38: 761-6.

3. Brigante G, Luzzaro F, Perilli M, et al. Evolution of CTX-M-type beta-lactamases in isolates of Escherichia coli infecting hospital and community patients. Int J Antimicrob Agents 2005; 25: 157-62.

4. Carattoli A, Lovari S, Franco A, Cordaro G, Di Matteo $\mathrm{P}$, Battisti A. Extended-spectrum beta-lactamases in Escherichia coli isolated from dogs and cats in Rome, Italy, from 2001 to 2003. Antimicrob. Agents Chemother 2005; 49: 833-5.

5. Luzzaro F, Mezzatesta M, Mugnaioli C, et al. Trends in production of extended-spectrum beta-lactamases among enterobacteria of medical interest: Report of the second Italian nationwide survey. J Clin Microbiol 2006; 44: 1659-64.

6. Pagani L, Dell'Amico E, Migliavacca R, et al.
Multiple CTX-M-type extended-spectrum beta-lactamases in nosocomial isolates of Enterobacteriaceae from a hospital in northern Italy. J Clin Microbiol 2003; 41: 4264-9.

7. Perilli M, Dell'Amico E, Segatore B, et al. Molecular characterization of extended-spectrum beta-lactamases produced by nosocomial isolates of Enterobacteriaceae from an Italian nationwide survey. J Clin Microbiol 2002; 40: 611-4.

8. Rice LB, Willey SH, Papanicolaou GA, et al. Outbreak of ceftazidime resistance caused by extended-spectrum ß-lactamases at a Massachusetts chronic-care facility. Antimicrob Agents Chemother 1990; 34: 2193-9.

9. Tenover FC, Arbeit RD, Goering RV, et al. Interpreting chromosomal DNA restriction patterns produced by pulsed-field gel electrophoresis: criteria for bacterial strain typing. J Clin Microbiol 1995; 33: 2233-9.

10. Tzouvelekis LS, Tzelepi E, Tassios PT, Legakis NJ. CTX-M-type beta-lactamases: an emerging group of extended-spectrum enzymes. Int J Antimicrob Agents 2000; 14: 137-42.

11. Wiener J, Quinn JP, Bradford PA. Multiple antibioticresistant Klebsiella and Escherichia coli in nursing homes. JAMA 1999; 281: 517-23. 